\title{
Distinguishing Epithelial Growth Factor Receptor Tyrosine Kinase Inhibitor induced Oral Mucositis/Stomatitis from Pseudomembranous Candidiasis: Case Series
}

\author{
Takashi Yurikusa, Kohei Furukawa \\ Division of Dentistry and Oral Surgery, Shizuoka Cancer Center, Shizuoka, Japan
}

\begin{abstract}
Introduction
Oral mucositis and stomatitis occurs quite frequently with some targeted therapies, namely epithelial growth factor receptor tyrosine kinase inhibitor (EGFR-TKI). The purpose of this report is to show the importance of careful consideration in the administration of antifungal medications through two case studies of EGFR-TKI-induced oral mucositis/stomatitis.
\end{abstract}

\section{Case presentation}

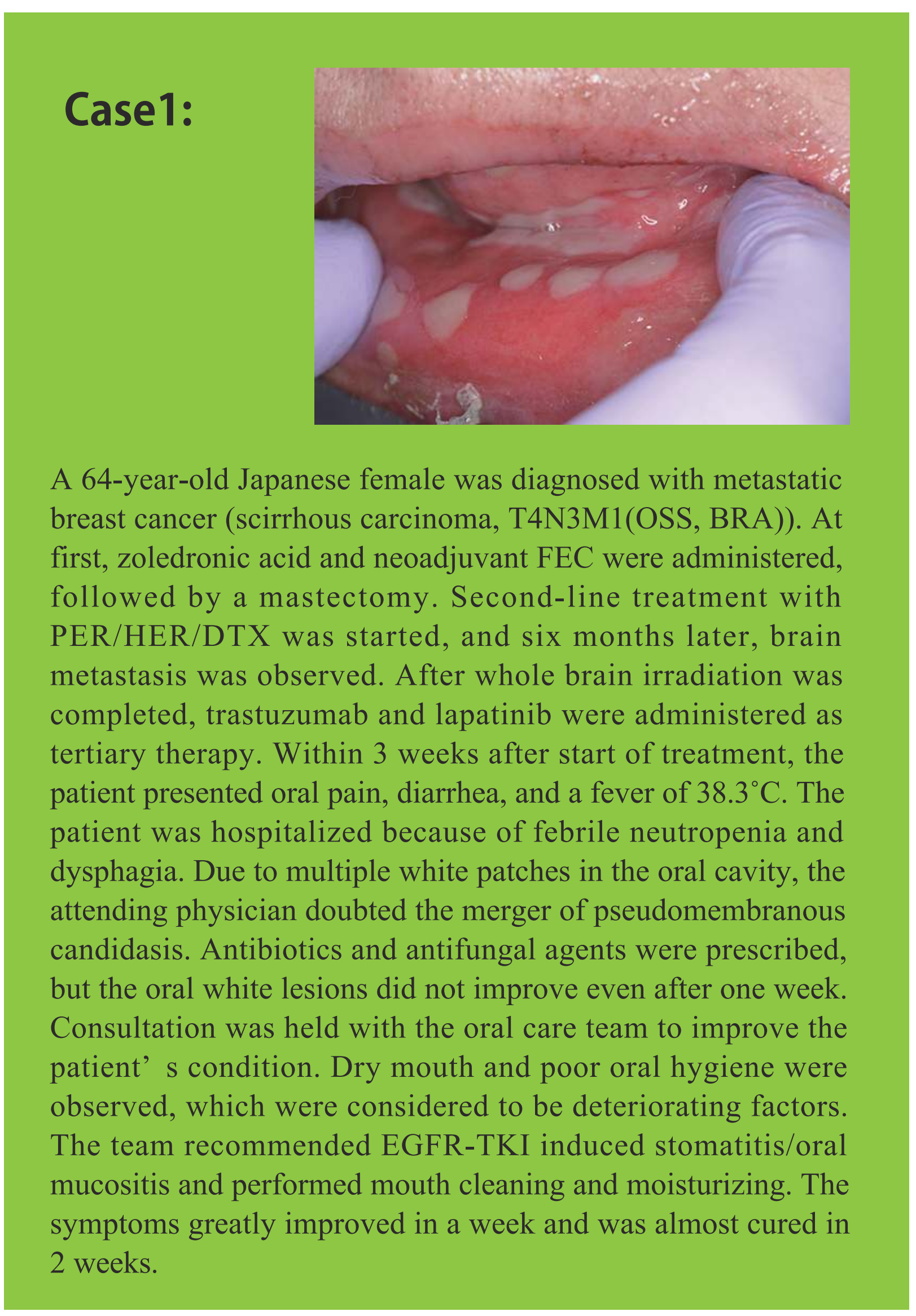

\section{Conclusion}

EGFR-TKI-induced oral mucositis/stomatitis must be distinguished from pseudomembranous candidiasis because drug interaction may prolong oral complications.

\section{Conflict of interest}

With regards to the theme of this e-poster presentation, there are no companies in COI relationship that should be disclosed.

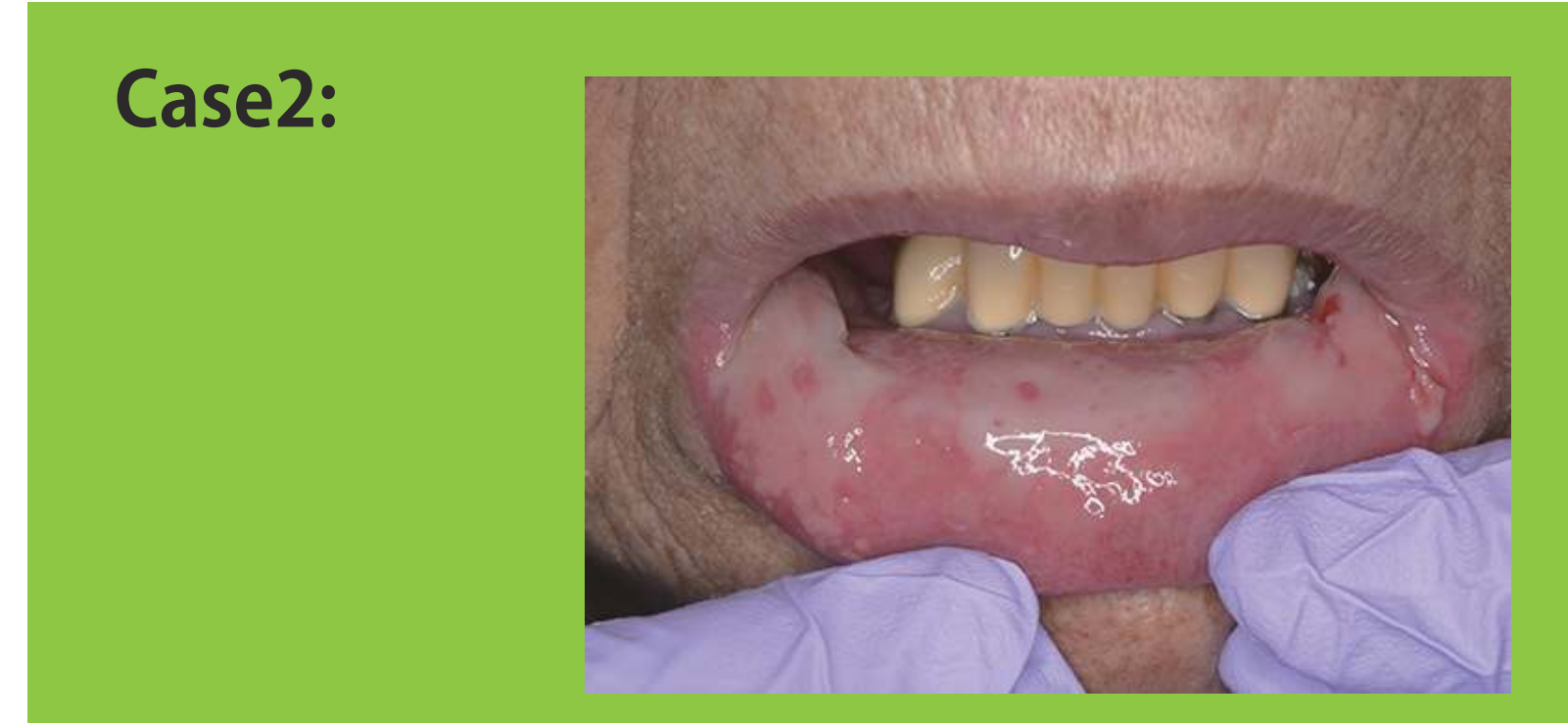

An 88-year-old Japanese female was diagnosed with metastatic non-small-cell lung cancer (adenocarcinoma, T3N2M1a(PM)). Mutational analysis of the tumor biopsy revealed the presence of an activation mutation (exon 19 deletion). First-line treatment with gefitinib was started and good partial response of the patient' s pulmonary tumors, but the primary tumor progressed after 2 years. Docetaxel was administrated as second-line treatment. Stabilization of the disease was obtained, but progression was confirmed after 1 year. Third-line treatment with re-gefitinib was performed, but again progression was confirmed after 1 year. A second biopsy was performed and T790M was revealed, then Fourth-line treatment with osimertinib was performed. In the 6 th week after the start of osimertinib, the patient complained of oral pain and lip swelling. There were multiple white lesions on the tongue, lower lip, cheeks, and soft plate. The patient had poor oral hygiene and couldn' $t$ use a toothbrush because of oral pain. The patient was instructed on how to use mouthwash with an anesthetic solution. An antifungal agent was prescribed, but the white lesions did not improve.

\section{Discussion}

The pathology of molecular targeted therapeutic agent related to stomatitis is still not yet well understood. Since mTOR inhibitor associated stomatitis (mIAS) shows the characteristics of aphthous stomatitis, topical corticosteroid seems to be effective and may also be used to prevent stomatitis. On the other hand, the pathology of EGFR-TKI-related stomatitis remains unknown. In some cases, it is reported that application of topical corticosteroids is effective as with skin rash. But stomatitis showing white patches, it is important to differentiate it from pseudomembranous Candida. The azole-based antifungal agents are metabolized by cytochrome P450 (CYP3A4) in the liver and may present drug interactions. It is known that some EGFR-TKI (gefitinib, erlotinib, lapatinib, osmertinib, etc.) are metabolized by CYP3A4, and metabolic inhibited by drug interaction may prolong oral mucosal disorder. If stomatitis develops while using EGFR-TKI, attention should be paid to the administration of antifungal medications. In these cases, EGFR-TKI treatment caused white patches in the oral cavity, so it was difficult to distinguish between stomatitis and pseudomembranous candida. 